University of Wollongong

Research Online

Faculty of Engineering and Information

Faculty of Engineering and Information

Sciences - Papers: Part A

Sciences

$1-1-2014$

\title{
Removing the impulsive noise contained in a self-mixing interferometry system using outlier detection
}

\author{
Yan Gao \\ University of Wollongong, yg904@uowmail.edu.au \\ Yanguang Yu \\ University of Wollongong, yanguang@uow.edu.au \\ Jiangtao Xi \\ University of Wollongong, jiangtao@uow.edu.au \\ Qinghua Guo \\ University of Wollongong, qguo@uow.edu.au \\ Jun Tong \\ University of Wollongong, jtong@uow.edu.au
}

See next page for additional authors

Follow this and additional works at: https://ro.uow.edu.au/eispapers

Part of the Engineering Commons, and the Science and Technology Studies Commons

\footnotetext{
Research Online is the open access institutional repository for the University of Wollongong. For further information
} contact the UOW Library: research-pubs@uow.edu.au 


\title{
Removing the impulsive noise contained in a self-mixing interferometry system using outlier detection
}

\begin{abstract}
Impulsive noise is a major problem that seriously degrades the performance of self-mixing interferometry (SMI). A new method to rectify this issue is proposed. First, an outlier detection approach is employed to detect the data samples corrupted by the impulsive noise, and then the SMI signal waveform is rectified by means of least square (LS) curve fitting. The results show that the proposed method can effectively remove the impulsive noise without introducing distortion to the original waveform and thus lead to improvement in the performance of an SMI system.
\end{abstract}

\section{Keywords}

interferometry, semiconductor laser, laser application

Disciplines

Engineering | Science and Technology Studies

\section{Publication Details}

Y. Gao, Y. Yu, J. Xi, Q. Guo, J. Tong \& S. Tong, "Removing the impulsive noise contained in a self-mixing interferometry system using outlier detection," Optical Engineering, vol. 53, (12) pp. 124108-1 - 124108-6, 2014.

\section{Authors}

Yan Gao, Yanguang Yu, Jiangtao Xi, Qinghua Guo, Jun Tong, and Sheng Tong 


\section{Optical Engineering}

\section{Removing the impulsive noise contained in a self-mixing interferometry system using outlier detection}

Yan Gao

Yanguang $\mathrm{Yu}$

Jiangtao Xi

Qinghua Guo

Jun Tong

Sheng Tong

\section{SPIE.}




\title{
Removing the impulsive noise contained in a self-mixing interferometry system using outlier detection
}

\author{
Yan Gao, Yanguang Yu, ${ }^{*}$ Jiangtao Xi, Qinghua Guo, Jun Tong, and Sheng Tong \\ University of Wollongong, Faculty of Engineering and Information Science, School of Electrical, Computer and Telecommunications, Northfields \\ Avenue, Wollongong, New South Wales 2522, Australia
}

\begin{abstract}
Impulsive noise is a major problem that seriously degrades the performance of self-mixing interferometry (SMI). A new method to rectify this issue is proposed. First, an outlier detection approach is employed to detect the data samples corrupted by the impulsive noise, and then the SMI signal waveform is rectified by means of least square (LS) curve fitting. The results show that the proposed method can effectively remove the impulsive noise without introducing distortion to the original waveform and thus lead to improvement in the performance of an SMI system. @ 2014 Society of Photo-Optical Instrumentation Engineers (SPIE) [DOI: 10.1117/1.OE.53.12.124108]
\end{abstract}

Keywords: interferometry; semiconductor laser; laser application.

Paper 141528 received Oct. 1, 2014; accepted for publication Dec. 2, 2014; published online Dec. 24, 2014.

\section{Introduction}

As a highly promising and emerging technique for noncontact sensing and measurement of semiconductor lasers (SLs) related parameters, the self-mixing interferometry (SMI) based on SLs has been an active field of research in recent years. ${ }^{1-5}$ The self-mixing interferometric effect occurs when a portion of light emitted by an SL is backscattered or reflected by an external target and re-enters the laser cavity, leading to modulations of the laser power both in amplitude and frequency. ${ }^{6}$ As the modulated output power, called an SMI signal, carries the information of both the target- and the SL-related parameters, ${ }^{7}$ there are two classes of applications for self-mixing based sensing: (1) estimation of parameters associated with an SL and (2) measurement of the metrological quantities of the external target. Many measurement algorithms have been reported for the above applications. ${ }^{2,5,8-16}$ The common procedure for implementing SMI-based sensing consists of steps including: (1) collecting the SMI signal using the experiment set-up illustrated in Fig. 1; (2) preprocessing the raw signal for the noise and the disturbance removal, and (3) parameter estimation.

The basic structure of an SMI system consists of, respectively, an SL laser source, an external target, and an electronic controlling part including the SL driving and SMI signal detecting devices. Generally, a single-mode SL is employed as the laser source to emit a laser beam onto an external target. The moving target is placed at the SL optical propagating path away from its front facet, which builds an external cavity for the SL. The light reflected by the external target re-enters the SL cavity and consequently modulates both the amplitude and frequency of the emitted lasing field. The modulated power, i.e., the SMI signal, is detected by a monitor photodiode (PD) packaged at the rear facet of the SL and the SMI signal is acquired by the signal detection unit.

A significant issue that impacts on the performance of SMI for applications mentioned above is the quality of the SMI signals. As seen from Fig. 1, an SMI system

*Address all correspondence to: Yanguang Yu, E-mail: yanguang@uow.edu.au contains both optical and electrical components; hence, the SMI signals inevitably contain noises due to the influence of various disturbances, e.g., the temperature fluctuation of the SL, background light, electronic noise, mode jumping during SL operation, and so on. The impulsive noise seriously degrades the performance of the applications as its existence leads to incorrect detections for SMI feature points to be used in existing measurement algorithms, ${ }^{2,5,8-16}$ such as the peak points, the bottom points, and the zero-crossing points. An example of the experimental SMI signal collected from the SMI system shown in Fig. 1 is depicted in Fig. 2. During the SMI signal collecting, the target is vibrating in a sinusoidal trajectory with a frequency of $70 \mathrm{~Hz}$ along the light axis. It should be noted that the SMI signal is a time-domain signal and $n$ is the discrete time index where the sampling frequency is $80 \mathrm{MHz}$. Traditional linear filtering can be used to reduce the noise, but they may also result in SMI waveform distortions. ${ }^{17,18}$ As most of the reported SMI-based sensing methods use the SMI waveform, the existence of such impulsive noise seriously degrades the performance of the SMI sensing. In this paper, we try to address this impulsive noise issue by means of a tailored signal processing approach for the SMI signal. Instead of using general linear filtering approaches, we propose to locate the impulsive noise by using outlier detection then applying appropriate curve fitting to rectify the waveform, and finally, applying an average filter.

\section{New Approach}

\subsection{Detection of Impulsive Noise}

According to Hawkins' definition, ${ }^{19}$ an outlier is an observation that deviates so significantly from other observations as to arouse suspicions. A number of approaches have been developed for outlier detection depending on different dataset characteristics, such as data distribution, whether the distribution parameters are known, the number of expected outliers and even the type of expected outliers. ${ }^{19}$ When an SMI system operates in a moderate/strong feedback, the 


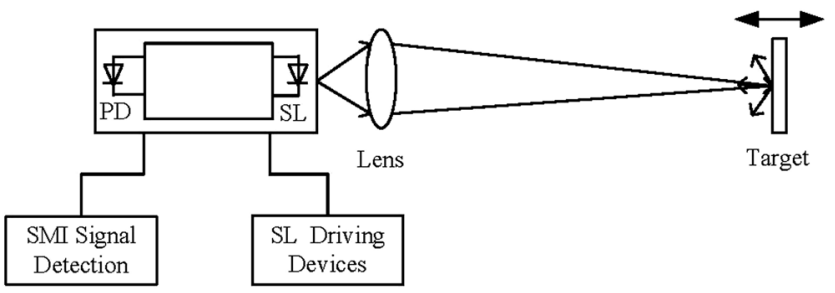

Fig. 1 Typical configuration of an SMI system, SL: semiconductor laser, PD: photo detector.

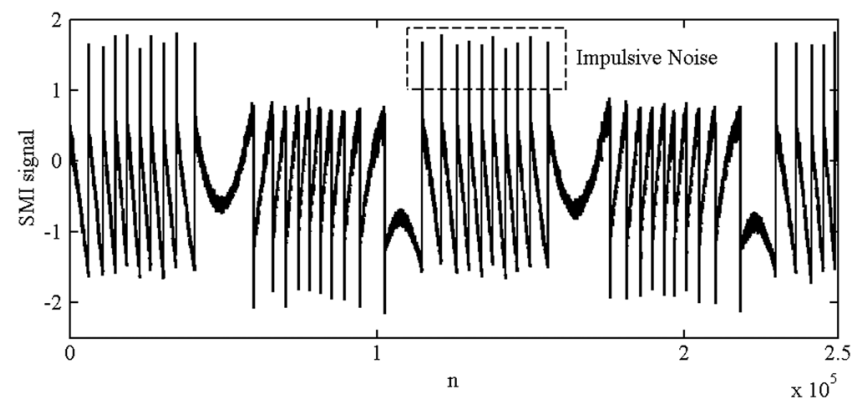

Fig. 2 Experimental SMI signal with large impulsive noise.

SMI signal is sawtooth-like. Figure 3(a) shows a piece of an experimental SMI signal containing four left declined fringes and two right inclined fringes. Figure 3(b) shows the enlarged picture of the fourth fringe marked in Fig. 3(a). The impulsive noise always occurs when the SMI signal exhibits a sharp jumping or dropping. Therefore, the signal samples corrupted by the impulsive noise can be treated as outliers among the normal SMI signal samples in the sawtooth-like waveform, which can be detected by the deviation-based outlier detection. ${ }^{20}$

Let us present the detailed procedure for locating the impulsive noise. In order to detect them, we divide the data into segments, each starting from the sharp change of the waveform and covering a whole individual fringe (i.e., to the next sharp change). Based on such a segmentation of the signal, we can ensure that the impulsive noise always occurs at the beginning of each segment. In the following, we first give the definitions for the data sets and functions used in our approach, and then present the proposed algorithm. For the easiness of manipulation, let us introduce the following notations:

- $X=[x(1), x(2), \ldots, x(N)]$ be a set of $N$ experimental data samples starting from the sharp change of the waveform and covering a whole individual fringe;

- $X_{j}=[x(1), x(2), \ldots, x(j)]$ be a subset of $X$, containing the first $j$ elements, where $j=1,2,3, \cdots, N-1$;

- $F=[f(1), f(2), \ldots, f(N)]$ be a standard reference function, representing the expected data set without the influence of the outliers; in the following, $F$ is obtained by Least Square (LS) fitting of $X$;

- $\bar{X}_{j}=[x(j+1), x(j+2), \ldots, x(N)]$, that is, the remaining elements of $X$ excluding $X_{j}$.

As the SMI signal in the moderate/strong feedback regimes has a saw-tooth like waveform, each segment of the signal is close to a straight line, and hence, we set the standard reference set as $f(n)=a_{0}+a_{1} n$, (for $n=1,2,3, \cdots, N)$. The two coefficients $a_{0}$ and $a_{1}$ can be

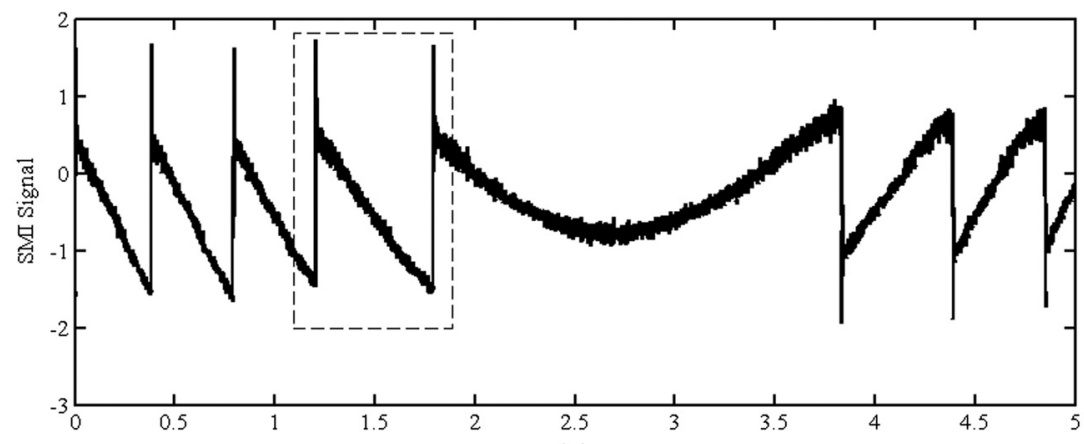

(a)

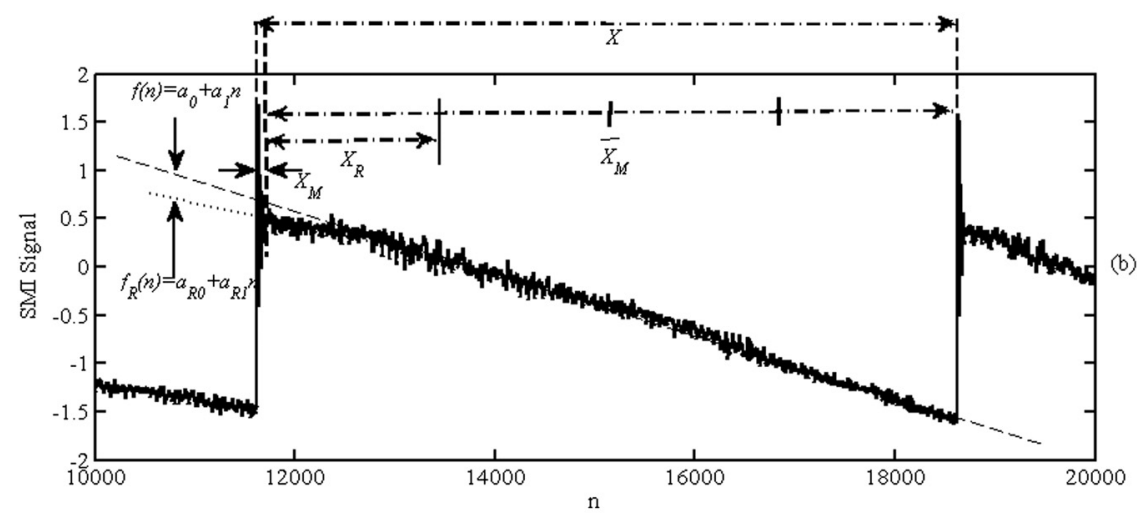

(b)

Fig. 3 Principle of outlier detection (a) a raw SMI signal and (b) enlarged view for a fringe. 
determined based on the actual data samples by means of LS curve fitting by minimizing the following objective function with respect to the two coefficients $a_{0}$ and $a_{1}$ :

$E\left(a_{0}, a_{1}\right)=\sum_{n=1}^{N}[x(n)-f(n)]^{2}$.

The optimal coefficients $a_{0}$ and $a_{1}$ can be determined by solving $\left[\partial E\left(a_{0}, a_{1}\right) / \partial a_{0}\right]=0$ and $\left[\partial E\left(a_{0}, a_{1}\right) / \partial a_{1}\right]=0$, yielding:

$$
\left(\begin{array}{l}
a_{0} \\
a_{1}
\end{array}\right)=\left\{\begin{array}{l}
\frac{\left(\sum_{n=1}^{N} n^{2}\right)\left[\sum_{n=1}^{N} X(n)\right]-\left[\sum_{n=1}^{N} X(n) \cdot n\right]\left(\sum_{n=1}^{N} n\right)}{N \sum_{n=1}^{N} n^{2}-\left(\sum_{n=1}^{N} n\right)^{2}} \\
\frac{N\left[\sum_{n=1}^{N} X(n) \cdot n\right]-\left(\sum_{n=1}^{N} n\right)\left[\sum_{n=1}^{N} X(n)\right]}{N \sum_{n=1}^{N} n^{2}-\left(\sum_{n=1}^{N} n\right)^{2}}
\end{array}\right\}
$$

Then a dissimilarity function can be defined to measure the difference between the experimental data and the standard reference function $F$. For the original data set $X$, the dissimilarity function is given by

$D(X)=\frac{1}{N} \sum_{n=1}^{N}[x(n)-f(n)]^{2}$

and for the data set excluding the subset $X_{j}$ we have

$D\left(\bar{X}_{j}\right)=\frac{1}{N-j} \sum_{n=j+1}^{N}[x(n)-f(n)]^{2}$.

Given that the outliers appear in the beginning of the data set, it is expected that $D\left(\bar{X}_{j}\right)$ decreases with $j$. In addition to the above, we also define the Smooth factor as below:
$S\left(X_{j}\right)=D(X)-D\left(\bar{X}_{j}\right)$

Note that $S\left(X_{j}\right)$ gives the difference between the original data set $X$ and its subset $X_{j}$ in terms of their dissimilarity (or difference) to the standard reference data set $F$. As the outliers appear in the beginning of the data set, with the increase of $j, S\left(X_{j}\right)$ should also increase when $X_{j}$ covers additional outliers; $S\left(X_{j}\right)$ should reach its maximum and remain stable if no additional outliers are covered by $X_{j}$. Based on such a scenario, we can check the value of $S\left(X_{j}\right)$ by increasing $j$, and when $S\left(X_{j}\right)$ reaches the maximum, we note the value of $j$ as $M$. The subset $X_{M}$ should contain all the outliers. In a mathematical language, $M$ is determined by

$S\left(X_{j}\right)<S\left(X_{M}\right), \quad 1<j<M-1$

and

$\left|S\left(X_{j}\right)-S\left(X_{M}\right)\right| \leq \delta, \quad M<j<N$,

where $\delta$ is a small positive tolerance for the experimental data fluctuations.

\subsection{Waveform Rectification}

With the procedure described above, the signal samples corrupted by the impulsive noise can be located. The next step is to repair them by replacing them with proper values. This can be done by curve fitting as well. As the impulsive noise occurs at the moderate/strong feedback and the SMI signals contain fringes of the saw-tooth like shape, we can use a linear fitting to replace the data samples that are corrupted. A simple and straight forward way is to use the standard reference data set $F$, i.e., $f(n)=a_{0}+a_{1} \cdot n$ built from set $X$. However, large errors will occur as seen from Fig. 3 . The dashed line represents the linear equation, which obviously cannot well fit the region of $X_{M}$. To solve this problem, we

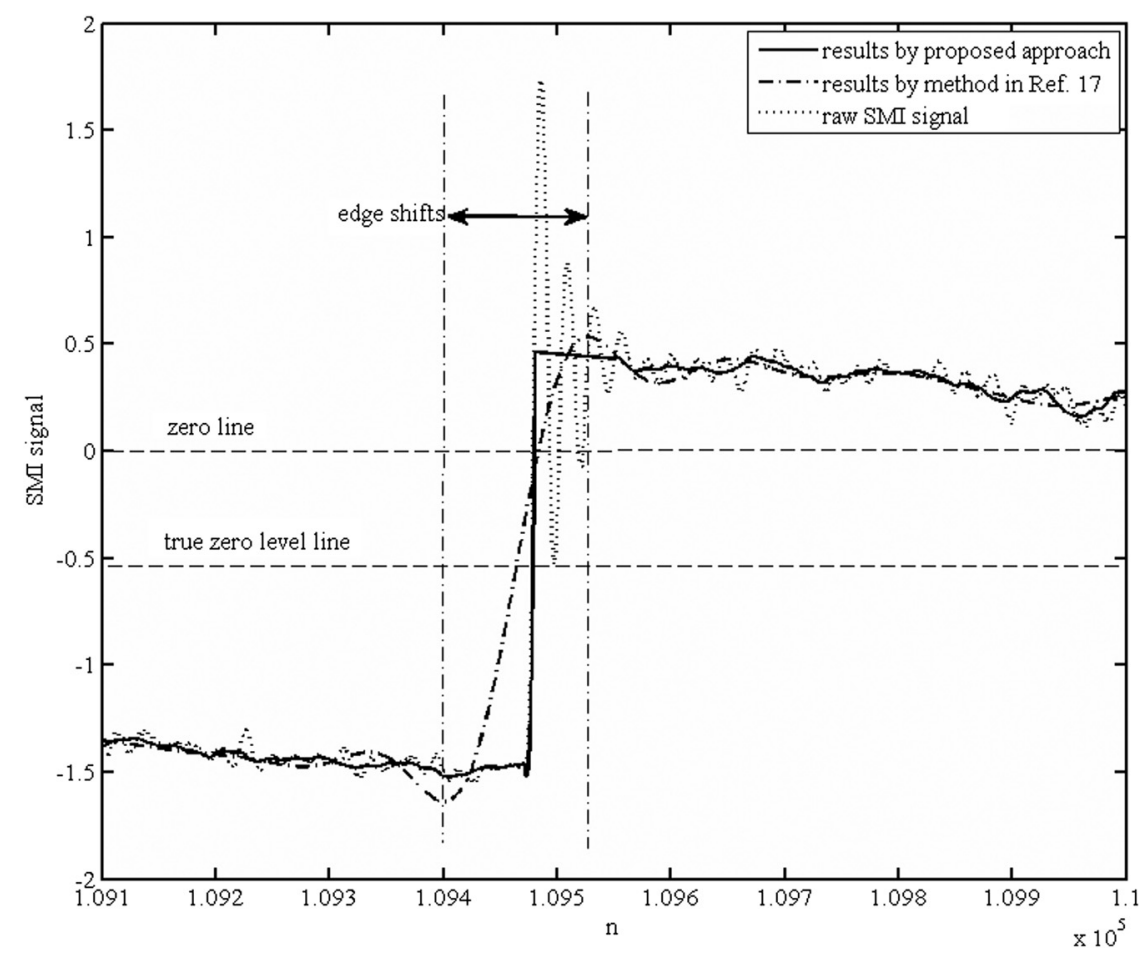

Fig. 4 Comparison the proposed approach with the method in Ref. 17. 


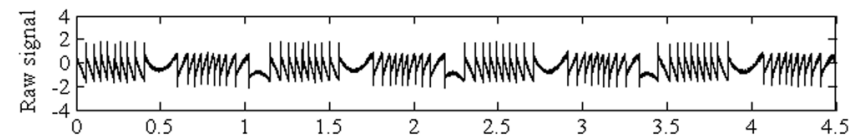

(a)

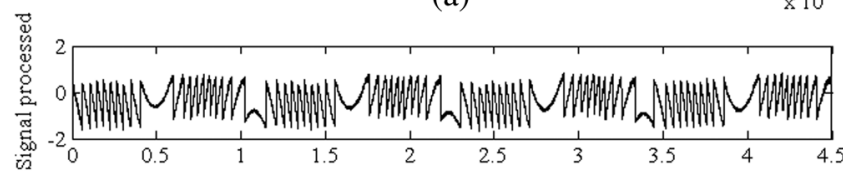

(b)

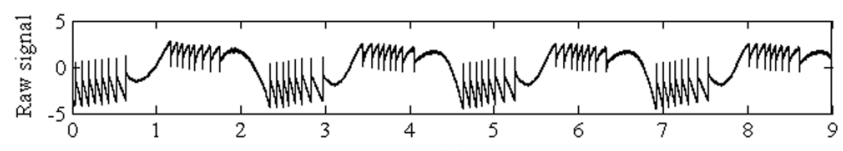

(c)

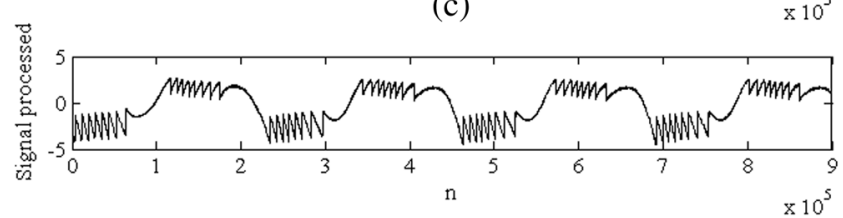

(d)

Fig. 5 Results processed by the proposed approach (a) raw SMI signal with harmonic vibration in moderate regime, (b) processed SMI signal in (a), (c) raw SMI signal with triangular vibration in strong regime, and (d) processed SMI signal in (c).

first remove the corrupted data samples and take a segment of samples (denoted as $X_{R}$ ) near the corrupted ones, where $X_{R}=[x(M+1), x(M+2), \ldots, x(M+R)]$. Based on $X_{R}$, we can do LS fitting to yield a straight line $F_{i R}$, i.e., $f_{R}(n)=$ $a_{R 0}+a_{R 1} \cdot n$ (dotted line shown in Fig. 3), which can be extended to cover the region of $X_{M}$. Since only the neighbor data samples are utilized, the extended data can better reflect the changing trend for the data samples within $X_{M}$. Choosing an $X_{R}$ too long or too short, we are not able to obtain the true trend of the fringe shape. Based on our observation of the experimental data, we found that one-quarter of the uncorrupted data samples is a suitable choice for the length of $X_{R}$, as shown in Fig. 3 .

We have presented a method to detect the impulsive noise in a piece of SMI signal and a way to rectify the waveforms. In practice, the above procedure may need to be repeated a few times when the number of outliers is significant. In the following, how to apply the proposed approach on an SMI experimental signal is summarized. First, we need to segment a piece of experimental SMI signal into fringes using the method described in Ref. 10. We use subscript $i$ to represent the data or data set related to the $i$ 'th fringe, e.g., $X_{i}$ for the $i$ 'th fringe.

The above can be implemented by the steps below:

Step 1: acquire a piece of the SMI signal and divide it into segments, yielding data set $X_{i}$;

Step 2: for each $X_{i}$, determine the standard reference function $F_{i}$ by means of LS fitting described by Eqs. (1) and (2);

Step 3: for $j=1,2, \cdots, M$, calculate dissimilarity functions using Eqs. (3) and (4), and smooth functions using Eq. (5);

Step 4: determine the value of $M$ by Eqs. (6) and (7), yielding the subset $X_{M}$ containing all the outliers;

Step 5: rectify the data set $X_{i}$ by replacing the data $X_{M}$ with $F_{i R}$;

Step 6: repeat the steps 2 to 5 for three to five times.
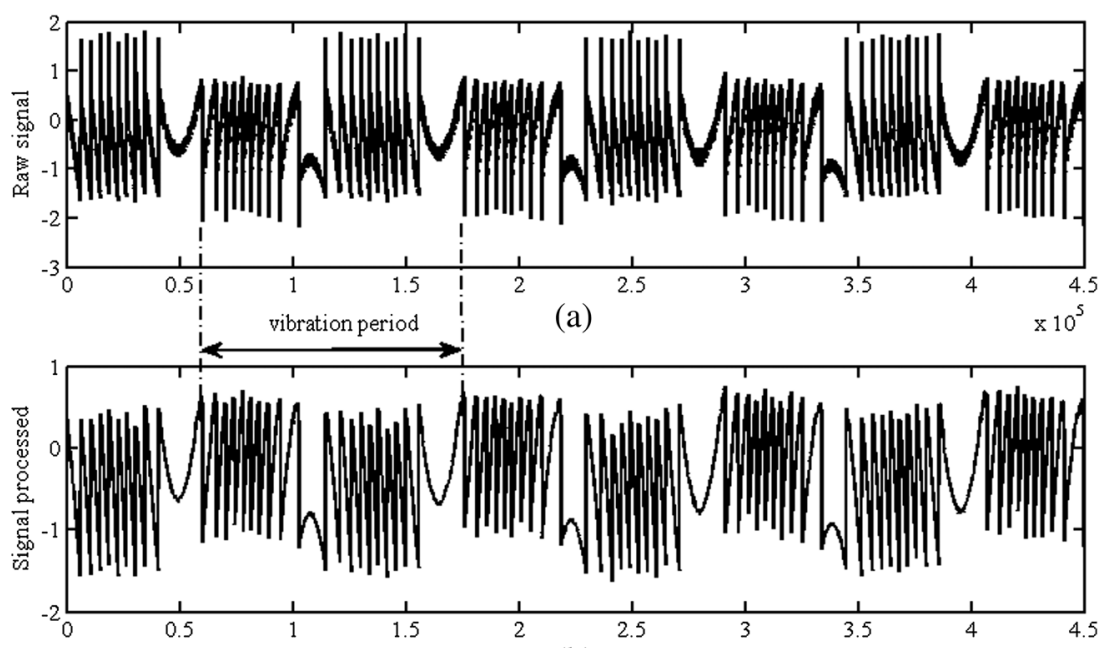

(b)

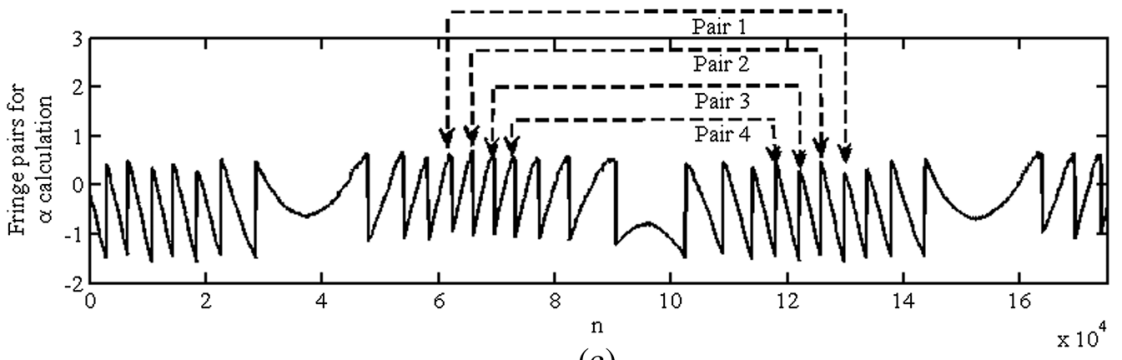

(c)

Fig. 6 Results processed by the proposed approach (a) raw SMI signal; (b) SMI signal processed by the proposed method; (c) fringe pairs for $\alpha$ estimating. 
Table 1 Estimation of $\alpha$.

\begin{tabular}{lcc} 
Method & $\begin{array}{c}\text { Average value } \\
(\hat{\alpha})\end{array}$ & $\begin{array}{c}\text { Maximum error } \\
(|\alpha-\hat{\alpha}|)\end{array}$ \\
\hline Method in Ref. 17 & 4.850 & 0.35 \\
Proposed method & 4.120 & 0.17 \\
\hline
\end{tabular}

\section{Verification}

Two pieces of the experimental SMI signals with large impulsive noise are obtained using the experiment setup illustrated in Fig. 1. The SL with a wavelength of $785 \mathrm{~nm}$ is biased with a dc current of $80 \mathrm{~mA}$ and operated at $25 \pm 0.1^{\circ} \mathrm{C}$. A loudspeaker is employed as the moving target which is driven by the signal generator. In order to verify the performance of the proposed approach, we first compared our filtering result with the one obtained by the approach reported in Ref. 17.

Figure 4 shows the comparison of details of a jumping edge occurring in between fringes. Two different experimental SMI signals are employed for verification. The first signal is collected with the harmonic vibration in a moderate feedback regime and the second one is collected with the triangular vibration in a strong feedback regime. Figure 5 shows two pieces of experimental SMI signals [Figs. 5(a) and 5(c)] and their filtering results by the proposed method [Figs. 5(b) and $5(d)$ ]. It can be seen from Fig. 4 that the proposed approach can remove the impulsive noise while keeping the jumping location nearly unchanged. However, the method in Ref. 17 introduces distortion for the jumping edge, i.e., smoothing the edge jumping area. Furthermore, we conducted the linewidth enhancement factor (denoted as $\alpha$ ) evaluation using the algorithm presented in Ref. 2. The estimation details are shown in Fig. 6. One pair of fringes within a vibration period of the external target can be used to work out the value of $\alpha$. For the experimental SMI signal obtained, we can choose four pairs of fringes within each vibration period as shown in Fig. 6(c). As the acquired experimental signal contains three vibration periods, we have 12 pairs of fringes which can generate 12 estimations of $\alpha$ in total. The average values of the all estimations and the maximum error are reported in Table 1. It can be seen that the measurement results of $\alpha$ from the proposed processing method are characterized by less deviation than that by the approach in Ref. 17. We noticed that the average results from the two approaches are different. However, as the proposed method leads to less distortion of the original SMI waveform, it is expected that the result (that is 4.120) obtained is closer to the true value of $\alpha$ than that from Ref. 17.

\section{Conclusion}

This paper presents a new preprocessing approach to improve the quality of SMI signal waveform corrupted by impulsive noise occurring in a moderate or strong optical feedback regime. The proposed approach is based on outlier detection and waveform rectification and can effectively remove the impulsive noise without introducing distortions to the original waveforms. Since SMI-based sensing and measurements rely significantly on the quality of the SMI signals, the proposed method is very effective for enhancing the measurement performance of the technology. It should be noted that the proposed method has no restriction on the movement of the target; hence, it can work for the case of a non-sinusoidal vibration.

\section{References}

1. G. Giuliani et al., "Laser diode self-mixing technique for sensing applications," J. Opt. A 4(6), S283-S294 (2002).

2. Y. Yu, G. Giuliani, and S. Donati, "Measurement of the linewidth enhancement factor of semiconductor lasers based on the optical feedback self-mixing effect," IEEE Photonics Technol. Lett. 16(4), 990992 (2004)

3. J. Xi et al., "Estimating the parameters of semiconductor lasers based on weak optical feedback self-mixing interferometry," IEEE J. Quant. Electron. 41(8), 1058-1064 (2005).

4. Y. Yu et al., "Toward automatic measurement of the linewidthenhancement factor using optical feedback self-mixing interferometry with weak optical feedback," IEEE J. Quant. Electron. 43(7), 527-534 (2007).

5. O. D. Bernal, U. Zabit, and T. Bosch, "Study of laser feedback phase under self-mixing leading to improved phase unwrapping for vibration sensing," IEEE Sensors J. 13(12), 4962-4971 (2013).

6. R. Lang and K. Kobayashi, "External optical feedback effects on semiconductor injection laser properties," IEEE J. Quant. Electron. 16(3), 347-355 (1980).

7. Y. Yu et al., "Optical feedback self-mixing interferometry with a large feedback factor: behavior studies," IEEE J. Quant. Electron. 45(7), 840-848 (2009).

8. S. Donati, G. Giuliani, and S. Merlo, "Laser diode feedback interferometer for measurement of displacements without ambiguity," IEEE J. Quant. Electron. 31(1), 113-119 (1995).

9. M. Norgia et al., "Frequency compensation for a self-mixing interferometer," IEEE Trans. Instrum. Meas. 59(5), 1368-1374 (2010).

10. Y. Fan et al., "Improving the measurement performance for a self-mixing interferometry-based displacement sensing system," Appl. Opt. 50 (26), 5064-5072 (2011).

11. S. Merlo and S. Donati, "Reconstruction of displacement waveforms with a single-channel laser-diode feedback interferometer," IEEE $J$. Quant. Electron. 33(4), 527-531 (1997).

12. N. Servagent, F. Gouaux, and T. Bosch, "Measurements of displacement using the self-mixing interference in a laser diode," J. Opt. 29(3), 168 (1998).

13. C. Bes, G. Plantier, and T. Bosch, "Displacement measurements using a self-mixing laser diode under moderate feedback," IEEE Trans. Instrum. Meas. 55(4), 1101-1105 (2006).

14. L. Wei et al., "Phase unwrapping of self-mixing signals observed in optical feedback interferometry for displacement measurement," in Int. Symp. on Intelligent Signal Processing and Communications, ISPACS '06, pp. 780-783, IEEE, Yonago (2006).

15. T. Bosch and S. Donati, "Distance and displacement measurements by laser technique," Opt. Eng. 40(1), 8-9 (2001).

16. M. Wang and G. Lai, "Displacement measurement based on Fourier transform method with external laser cavity modulation," Rev. Sci. Instrum. 72(8), 3440-3445 (2001).

17. Y. Yu, J. Xi, and J. Chicharo, "Improving the performance in an optical feedback self-mixing interferometry system using digital signal preprocessing," in IEEE Int. Symp. on Intelligent Signal Processing, pp. 1-6, IEEE, Alcala de Henares (2007).

18. L. Wei et al., "Pre-processing of signals observed from laser diode selfmixing intereferometries using neural networks," in IEEE Int. Symp. on Intelligent Signal Processing, pp. 1-5 (2007).

19. E. M. Knorr, R. T. Ng, and V. Tucakov, "Distance-based outliers: algorithms and applications," VLDB J. 8(3-4), 237-253 (2000).

20. A. Andreas, A. Rakesh, and R. Prabhakar, "A linear method for deviation detection in large database," in Proc. KDD-96, pp. 164 169 (1996).

Yan Gao is a PhD candidate at the University of Wollongong. She received a $\mathrm{BE}$ degree in electrical engineering from Zhengzhou University, China, in 2010.

Yanguang $\mathbf{Y u}$ is a senior lecturer at the University of Wollongong, Australia. She received $\mathrm{BE}$ and $\mathrm{PhD}$ degrees, respectively, from Huazhong University of Science and Technology in 1986 and Harbin Institute of Technology in 2000 in China. Her current research interests include optoelectronic systems, 3-D profile measurement, and telecommunication systems.

Jiangtao $\mathbf{X i}$ is a professor at the University of Wollongong. He received a $\mathrm{BE}$ degree from Beijing Institute of Technology, China, in 1982, an ME degree from TsingHua University, China, in 1985, 
and a PhD degree from the University of Wollongong, Australia, in 1996, all in electrical engineering. His current research interests include signal processing with applications to instrumentation and measurements, telecommunications, and optoelectronics systems. $\mathrm{He}$ is a senior member of IEEE and a member of SPIE.

Qinghua Guo is a senior lecturer at the University of Wollongong. He received a $B E$ degree in electronic engineering, an ME degree in signal and information processing from Xidian University in 2001 and 2004, respectively, and a PhD degree in electronic engineering from the City University of Hong Kong in 2008. He was a recipient of the Australian Research Council's Inaugural Discovery Early Career Researcher Award.
Jun Tong is a lecturer at the University of Wollongong, Australia. He received $\mathrm{BE}$ and $\mathrm{ME}$ degrees from the University of Electronic Science and Technology of China, in 2001 and 2004, respectively, and the PhD degree from City University of Hong Kong in 2009. His research interests include signal processing and telecommunication systems.

Sheng Tong is a lecturer with the University of Wollongong and an associate professor with Xidian University. He received his BE, ME, and PhD degrees from Xidian University, Xi'an, China, in 2001, 2004, and 2006, respectively. His research interests include channel coding and iterative receiver design in digital communication systems. 